\title{
NFIB is an Epithelial Stem Cell Regulator of Melanocyte Stem Cells in the Hair Follicle Niche
}

\author{
H. Amalia Pasolli, Chiung-Ying Chang and Elaine Fuchs
}

Howard Hughes Medical Institute, Laboratory of Mammalian Cell Biology and Development, The Rockefeller University, 1230 York Avenue, New York, NY 10065.

In mammals, cyclical bouts of hair regeneration and pigmentation are fueled by a niche ("the bulge") that contains both hair follicle (HF) and melanocyte (Mc) stem cells (SCs). Through complex three-way crosstalk that involves specialized mesenchymal cells ("dermal papilla", DP) at the niche base and signaling through Wnts and TGF $\beta$ s, synchrony is achieved such that a few SCs of each type will initiate lineage commitment simultaneously as the new hair cycle begins. Coordinated activity continues throughout the hair cycle: during the growth phase (anagen), melanocytes in the hair bulb produce and transfer pigment to adjacent HFSC progeny (matrix) as they stop proliferating and begin to differentiate into hair (anagen)); as the destructive phase (catagen) ensues, melanocytes and matrix cells simultaneously undergo apoptosis, the DP is drawn upward and the follicle returns to a resting state (telogen). Dissecting the molecular crosstalk that governs this intricate tango has been difficult, since mutations that affect one lineage typically affect the other.

In studying the functional importance of a transcription factor that is highly expressed in HFSCs, we unexpectedly uncoupled this synchrony. Without NFIB, HFSCs behaved surprisingly normally, executing a proper hair cycle and follicle architecture. By contrast, in the quiescent stem cell niche, wild-type McSCs proliferated, and those close to the DP differentiated. Digging deeper into mechanism, we performed transcriptional profiling and NFIB ChIP-seq analyses on HFSCs, and conducted further functional studies. The prime DP stimulant appeared to be Kit-Ligand. Edn2 surfaced as a direct NFIB target gene whose transcription and encoded protein were dramatically elevated in NFIBdeficient HFSCs. Ectopic endothelin-2 recapitulated the NFiB-deficient phenotype in otherwise wild-type HFSCs, and an endothelin-receptor antagonist prevented precocious McSC proliferation and differentiation in the Nfib-null HFSC niche. Implicated in neural crest specification of the melanocyte lineage, endothelins have not been studied in the context of stem cell biology. Our findings provide new insights into how McSC and HFSC behaviors maintain reliance upon cooperative factors within the niche and how this might be uncoupled in injury and disease states.

Chang C-Y, Pasolli HA, Giannopoulou EG, Guasch G, Gronostajski RM, Elemento O and Fuchs E.NFIB is a governor of epithelial-melanocyte stem-cell behavior in a shared niche. Nature 2013, in press. 


\section{Control}

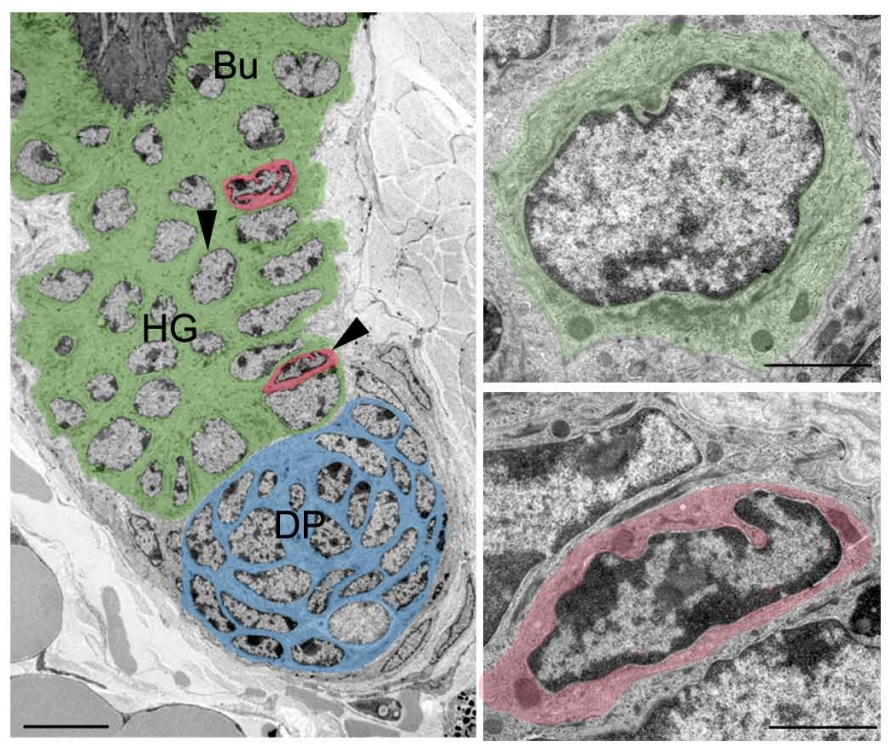

NFIB cKO
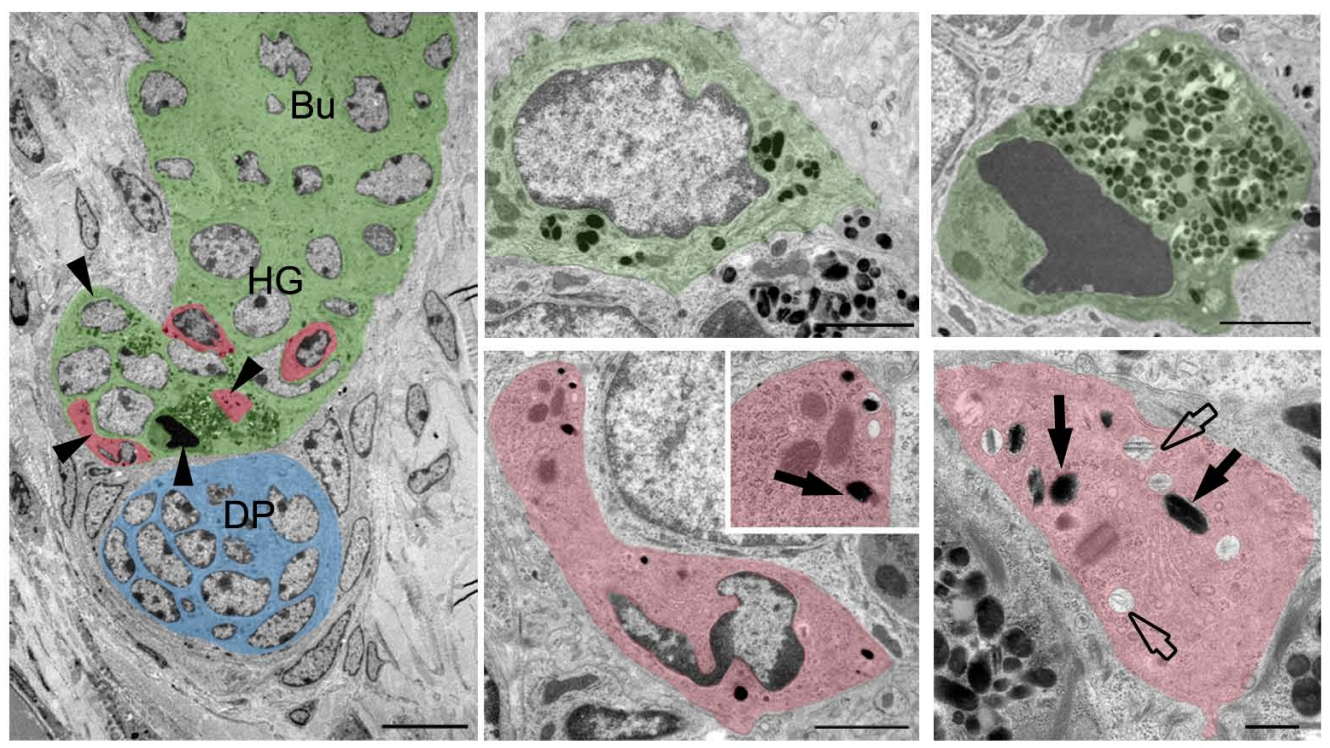

NFIB loss enhances melanocyte stem-cell self-renewal and perturbs melanocyte stem-cells activity in the hair follicle stem cell niche.

Hair follicle ultrastructure. There is an increased number of melanocytes and ectopic differentiation (as noticed by the presence of melanin granules) in telogen $\mathrm{cKO}$ hair follicles. Pseudo-colouring highlights distinct stem cell niche cell types: green, hair follicle stem cell; red, melanocyte; blue, dermal papilla. Arrowheads mark cells shown at higher magnification. Open and solid arrows denote immature and mature melanosomes, respectively. Note pigment-laden apoptotic hair follicle stem cells whose boxed regions at higher magnification reveal condensed chromatin, degenerating mitochondrion, keratin filaments (Kf), desmosomes (De) and melanosomes. Scale bars: $10 \mu \mathrm{m}$ (low magnification frames) $2 \mu \mathrm{m}$ all the other frames. 